〈红外应用〉

\title{
直埋热管道红外伪装技术研究
}

\author{
苏荣华 ${ }^{1}$, 刘定文 ${ }^{2}$, 李 锋 $^{1}$, 吴文明 ${ }^{1}$, 李玉鹏 ${ }^{1}$, 徐明新 ${ }^{1}$ \\ (1. 军事科学院 国防工程研究院, 北京 100850；2. 军事科学院 军事医学研究院，北京 100039）
}

\begin{abstract}
摘要: 随着现代红外技术的不断成熟和日榛完善, 直埋热管道面临的红外侦察监视和制导武器的威胁 日益增大, 为此必须加强直埋热管道的热红外伪装技术研究。本文利用传热学原理, 通过建立直埋热 管道温度的数值模型, 分析了管道埋深、冷热流体混合和包覆保温材料等措施对热红外伪装效果的影 响, 分析结果对直埋热管道的热红外伪装设计具有重要的参考价值。
\end{abstract}

关键词: 直埋热管道; 数值模型; 红外伪装

中图分类号：TB131 文献标识码：A 文章编号：1001-8891(2020)07-0702-05

\section{Research on Infrared Camouflage Technology for Direct Buried Heat Pipelines}

\author{
SU Ronghua ${ }^{1}$, LIU Dingwen ${ }^{2}$, LI Feng ${ }^{1}$, WU Wenming ${ }^{1}$, LI Yupeng ${ }^{1}$, XU Mingxin ${ }^{1}$ \\ (1. Research Institute for National Defense Engineering of Academy of Military Sciences PLA China, Beijing 100850, China; \\ 2. Academy of Military Medical Sciences, Beijing 100039, China)
}

\begin{abstract}
The threats of infrared reconnaissance, surveillance, and guidance weapons to directly buried heat pipelines are increasing with the development of modern infrared technology. Therefore, it is necessary to conduct extensive research on thermal infrared camouflage technology for directly buried heat pipelines. Based on the principle of heat transfer, this paper establishes a numerical model of the temperature of the directly buried heat pipe and analyzes the influence of the buried depth of the pipe, the mixing of cold and hot fluids, and the coating of thermal insulation materials on the thermal infrared camouflage effect. The analysis results provide an important reference for the thermal infrared camouflage design of the directly buried heat pipe.
\end{abstract}

Key words: direct buried heat pipeline, numerical model, infrared camouflage

\section{0 引言}

输油管道、高温气体排放管道等直埋热管道是地 表附近的一个异常热源, 在红外成像图上能清晰地观 察到直埋热管道所代表的一条亮线, 从而引起红外暴 露。用红外热像仪对直埋热管道进行探测 ${ }^{[1]}$, 结果如 图 1 所示。

直埋热管道红外暴露特征主要是热流体、管道壁、 土壤和空气之间对流、热传导和辐射换热引起的地表 辐射温差。由平面来看, 管道呈线状, 由断面来看, 排烟管道呈点状。埋管的土质如果较均匀一致, 管道 断面辐射方向的温度梯减也比较有规律。热扩散到地 面, 等温线被地表切断, 形成热管上方温度最高, 而
离管道轴线越远温度越低的现象, 形成了一条直埋热 管道上的温差带, 从而导致了埋地管道的红外暴露。

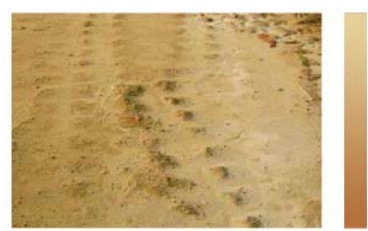

(a) 可见光图像

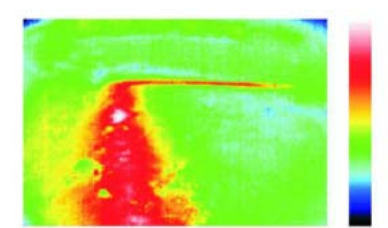

(b) 红外伪彩色图像
(a) Visible image
(b) Infrared pseudo color image

图 1 直埋热管道可见光与红外图像

Fig.1 Visible and infrared images of direct buried heat pipeline

直埋热管道热红外伪装目的包括 3 个方面: 一是 裸露地表背景条件下, 控制地表的辐射温差; 二是植 
被背景条件下, 还要控制管道外壁及土壤中的温度, 使其不大于 $50^{\circ} \mathrm{C}$, 以保证植物根系的成活; 三是控制 热管道出口的温度。其伪装措施主要包括采用不同管 道材质、增加埋深、冷热流体混合和包覆保温材料等。 文献[2-5]利用有限元法分别研究了埋地热管道不同 季节、不同地貌的土壤温度场和沿程温降情况, 提出 了大量有价值的求解方法。下面通过建立直埋热管道 的数值模型, 分析不同的伪装措施对直埋热管道热红 外伪装效果的影响。

\section{1 物理模型}

为简化计算模型, 做以下简化: (1)忽略大气温度 变化对埋地管道传热的影响; (2)忽略土壤中因水分迁 移而引起的热迁移, 认为埋管与土壤之间只是通过纯 导热进行传热; (3)埋管与土壤完好接触, 忽略接触热 阻; (4)认为土壤是均质的、导热系数不变; (5)在距管 道埋深横向方向 $16 \mathrm{~m}$ 处, 不考虑水平方向的热流。

建立如图 2 所示的直埋热管道物理模型: 其中管 道埋深 $0.5 \mathrm{~m}$, 管外径 $1.15 \mathrm{~m}$, 管道壁厚 $0.3 \mathrm{~m}$, 管长 $20 \mathrm{~m}$, 土壤深度 $8 \mathrm{~m}$, 土壤宽度 $32 \mathrm{~m}$ 。

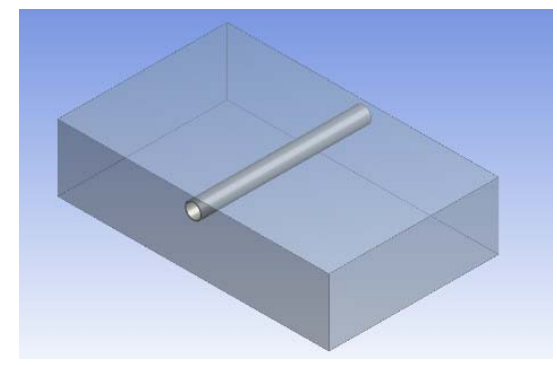

图 2 直埋热管道简化模型

Fig.2 Simplified model of direct buried heat pipeline

\section{2 数值模型}

热流体在直埋管道中传输时, 会与四周土壤进行 热交换，直埋热管道与周围环境的热交换大致分为 4 个过程: (1)热流体与管道的强制对流换热; (2)管道各 层的热传导; (3)管道外壁与周围土壤之间的热传导; (4)地表与大气之间的对流换热 ${ }^{[6]}$ 。

1）土壤表面与空气接触, 它们之间的热交换形 式主要是对流换热, 为第三类边界条件。用公式表示 为:

$$
-\left.\frac{\partial T}{\partial n}\right|_{\Gamma}=\left.\alpha\left(T-T_{\mathrm{f}}\right)\right|_{\Gamma}
$$

式中: $\alpha$ 为地表与空气的对流换热系数, $\mathrm{W} /\left(\mathrm{m}^{2} \cdot{ }^{\circ} \mathrm{C}\right)$; $T$ 为地表温度, ${ }^{\circ} \mathrm{C} ; T_{\mathrm{f}}$ 为空气温度, ${ }^{\circ} \mathrm{C} ; \Gamma$ 为地表与空 气接触面。

2）土壤与管道外表面接触, 它们之间的热交换
主要是热传导, 为第二类边界条件。用公式表示为:

$$
-\left.k \frac{\partial T}{\partial n}\right|_{\Gamma}=q
$$

式中: $q$ 为热流密度, $\mathrm{W} / \mathrm{m}^{2} ; k$ 为管道最外壁的导热 系数, $\mathrm{W} /\left(\mathrm{m} \cdot{ }^{\circ} \mathrm{C}\right) ; \Gamma$ 为管道外壁与土壤的接触面。

3）管道内表面与热介质接触，它们之间的热交 换形式主要是强制对流换热，为第三类边界条件。用 公式表示为:

$$
-\left.\frac{\partial T}{\partial n}\right|_{\Gamma}=\left.\alpha\left(T-T_{y}\right)\right|_{\Gamma}
$$

式中: $\alpha$ 为管道内表面与热流体的强制对流换热系数, $\mathrm{W} /\left(\mathrm{m}^{2} .{ }^{\circ} \mathrm{C}\right) ; T$ 为管道内壁温度, ${ }^{\circ} \mathrm{C} ; T_{y}$ 为高温烟气的 温度, ${ }^{\circ} \mathrm{C} ; \Gamma$ 为地表与空气接触面。

4）地表以下 $8 \mathrm{~m}$ 深度处的水平面, 为第一类边 界条件。用公式表现为:

$$
T_{\Gamma}=T_{\mathrm{o}}
$$

式中: $T_{\Gamma}$ 为地下 $8 \mathrm{~m}$ 深处土壤的温度, ${ }^{\circ} \mathrm{C} ; T_{0}$ 为大地 恒温层温度, 根据大地温度场实际测量结果, 把 $8 \mathrm{~m}$ 定为恒温层, 温度为 $4^{\circ} \mathrm{C}$ 。

5) 土壤左右两侧 $16 \mathrm{~m}$ 处的垂直面简化为绝热面。 用公式表现为:

$$
-\left.k \frac{\partial T}{\partial n}\right|_{\Gamma}=q
$$

式中: $q$ 为热流密度 $q=0 \mathrm{~W} / \mathrm{m}^{2} ; k$ 为土壤的导热系数, $\mathrm{W} / \mathrm{m} \cdot{ }^{\circ} \mathrm{C}$; 其它参数的设置: 气温为 $-20^{\circ} \mathrm{C}$, 自然对流 换热系数 ${ }^{[5]}$ 为 $20.74 \mathrm{~W} /\left(\mathrm{m}^{2} \cdot{ }^{\circ} \mathrm{C}\right)$, 热流体介质初始温度 $300^{\circ} \mathrm{C}$, 流速 $2 \mathrm{~m} / \mathrm{s}$, 管道密度 $2500 \mathrm{~kg} / \mathrm{m}^{3}$, 导热系数 $1.7 \mathrm{w} /\left(\mathrm{m} \cdot{ }^{\circ} \mathrm{C}\right)$, 其计算结果如图 3 所示。

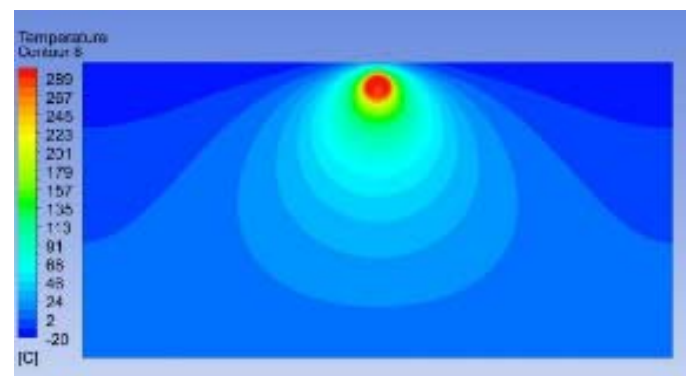

(a) 横断面 (a) Cross section

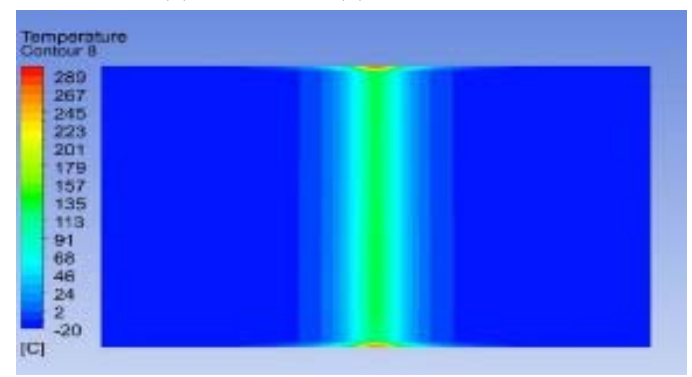

(b) 地表平面 $\quad$ (b) Surface plane

图 3 直埋热管道及土壤的温度分布图

Fig.3 Temperature distribution of direct buried heat pipes and soil 


\section{3 不同伪装对策时的数值模拟与分析}

\section{1 管道埋深对伪装效果的影响分析}

根据 CJJT81《城镇直埋热管道工程技术规程》规 定直埋热管道埋深不低于 $0.6 \mathrm{~m}$, 以及绿化伪装覆土 厚度不低于 $0.5 \mathrm{~m}$ 的要求, 我们分别对 $0.5 \mathrm{~m} 、 1 \mathrm{~m} 、 2$ $\mathrm{m} 、 3 \mathrm{~m}$ 四个不同埋深条件下的土壤表面温度、土壤 内温度分布和热管道内流体温度分布进行计算, 其结 果如图 4、图 5、图 6 所示。

地表温度受管道埋深影响较大, 由图 4 可以看出 随着管道埋深的增加, 地表最大温差和温差大于 $4^{\circ} \mathrm{C}$ 的地表宽度都在逐渐减小, 这对直埋热管道热红外伪 装是有利的, 当管道埋深达到 $3 \mathrm{~m}$ 时, 其地表最大温 差为 $3^{\circ} \mathrm{C}$ 。对地表最大温差 $\Delta T$ 和管道埋深 $s$ 的关系进 行了拟合, 得出以下拟合公式。

$$
\Delta T=23.215 \mathrm{e}^{0.476 s}
$$

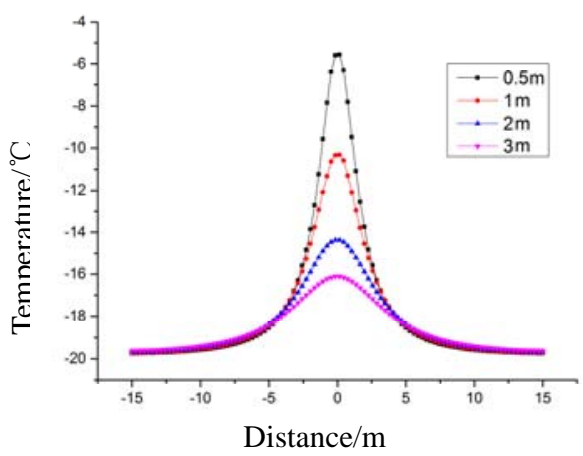

图 4 直埋热管道不同埋深时的地表温度分布

Fig.4 Surface temperature distribution at different buried depths of direct buried heat pipelines

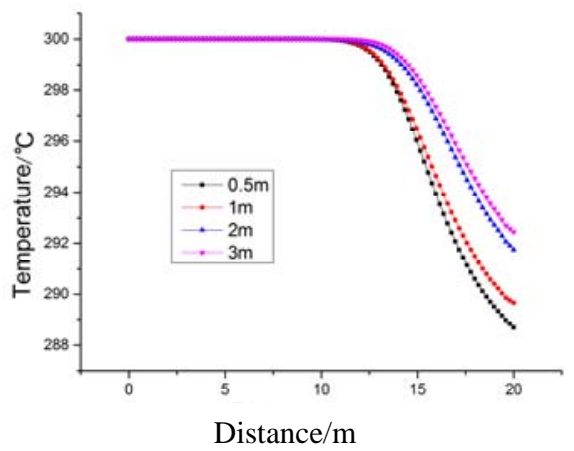

图 5 直埋热管道不同埋深时的流体温度分布

Fig.5 Fluid temperature distribution at different buried depths of direct buried heat pipelines

由图 5 可以看出, 随着管道埋深的增加, 管道出 口流体温度上升, 但即便是埋深 $0.5 \mathrm{~m}$ 的时候, 其流 体出口温度也达 $288.68^{\circ} \mathrm{C}$, 所以对管道出口的热红外 伪装要采取其他措施处理; 由图 6 可以看出, 随着管 道埋深的增加, 土壤中温度高于 $50^{\circ} \mathrm{C}$ 区域在增加, 这
对植物伪装是不利的。

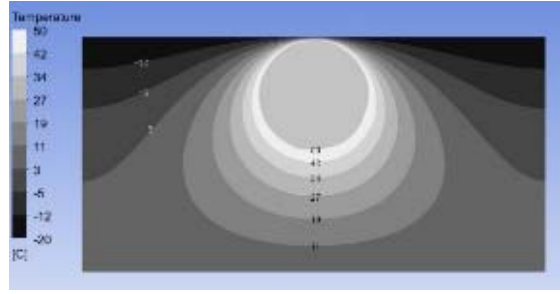
(a) $0.5 \mathrm{~m}$ 埋深
(a) $0.5 \mathrm{~m}$ buried depth

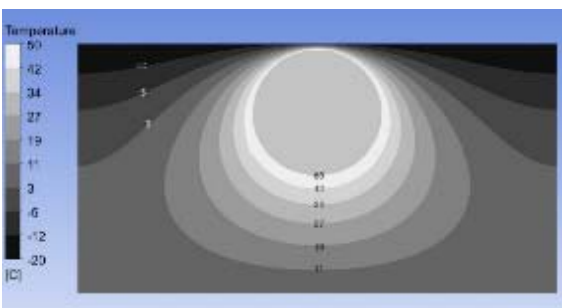

(b) $1 \mathrm{~m}$ 埋深

(b) $1 \mathrm{~m}$ buried depth

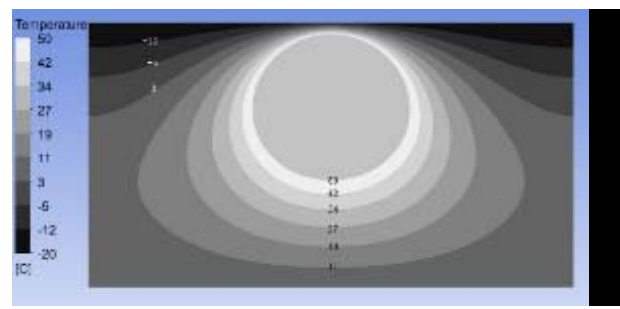

(c) $2 \mathrm{~m}$ 埋深 (c) $2 \mathrm{~m}$ buried depth

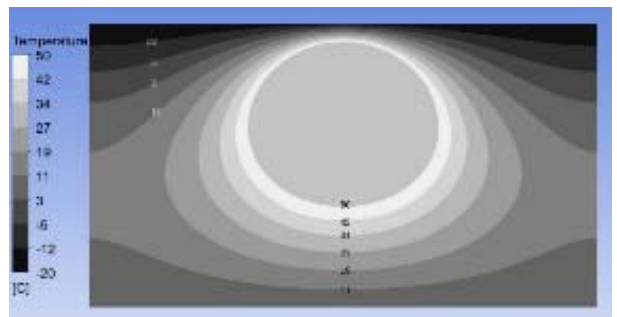

(d) $3 \mathrm{~m}$ 埋深 (d) $3 \mathrm{~m}$ buried depth

图 6 不同埋深时土壤内的温度分布

Fig.6 Temperature distribution in soil at different burial depths

\section{2 冷热流体混合对伪装效果的影响分析}

利用冷流体与管道内的高温流体混合来降低管 道和管道出口的温度, 是常用的红外伪装方法。冷热 流体的混合比例一方面取决于红外伪装对管壁温度 的要求, 另一方面取决于冷流体的储存量。我们采用 高温流体与 $20^{\circ} \mathrm{C}$ 的常温同质流体按不混合、1:1、1:2 和 1:4 四种比例混合，对其土壤表面温度、土壤温度 分布进行了计算, 分析对红外伪装效果的影响, 其结 果如图 7、图 8、图 9 所示。

通过采用常温流体与热流体混合的伪装措施，一 方面降低管道内流体的温度, 另一方面提高流度。由 图 7 可以看出, 不混合、冷热流体 1:1 混合、冷热流 体 2:1 混合和冷热流体 $4: 1$ 混合, 4 种情况下地表最大 温差 $\Delta t$ 分别为 $14.27^{\circ} \mathrm{C} 、 9.78^{\circ} \mathrm{C} 、 7.8^{\circ} \mathrm{C} 、 5.9^{\circ} \mathrm{C}$, 温差 
大于 $4^{\circ} \mathrm{C}$ 宽度分别为 $5.14 \mathrm{~m} 、 3.94 \mathrm{~m} 、 2.72 \mathrm{~m} 、 1.51 \mathrm{~m}$ 。 对地表最大温差 $\Delta T$ 和混合比例 $b$ 的关系进行了拟合, 得出以下拟合公式:

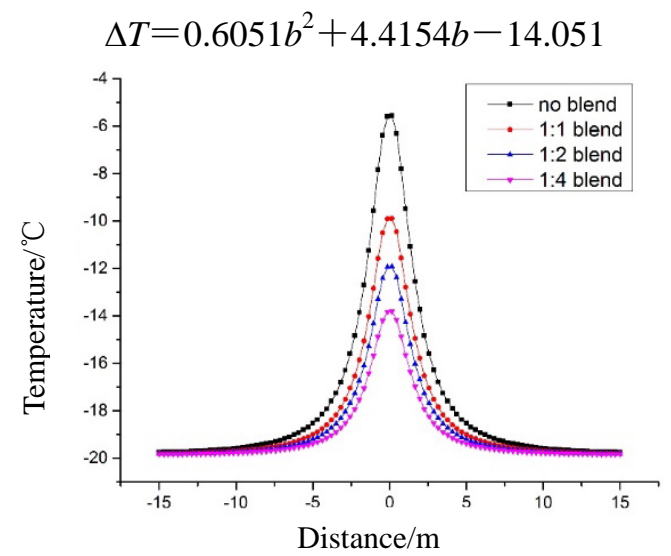

图 7 不同比例冷热流体混合时直埋热管道的地表温度分布

Fig.7 Surface temperature distribution of direct buried heat pipes when cold and hot fluids are mixed in different proportions

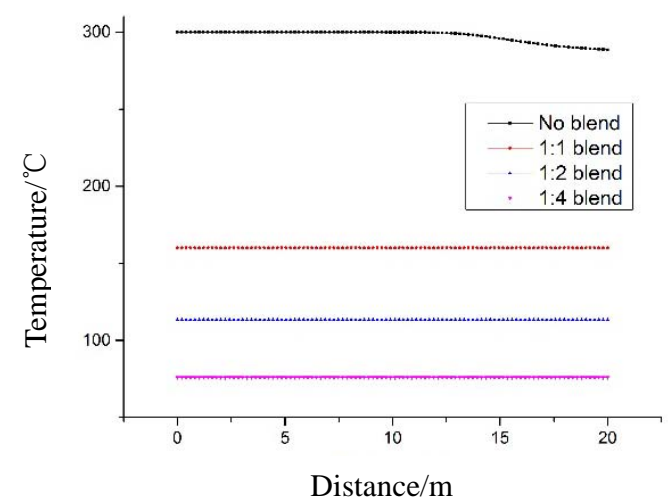

图 8 不同比例冷热流体混合时直埋热管道的流体温度分布

Fig.8 Fluid temperature distribution of direct buried heat pipes when cold and hot fluids are mixed in different proportions

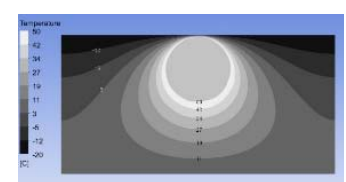

(a) 无常温流体混合

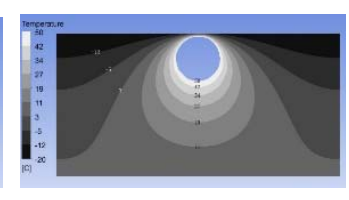

(b) 与常温流体 $1: 1$ 混合 (a) No hot and cold fluid mixing $\quad$ (b) 1:1 mixing of cold and hot fluid
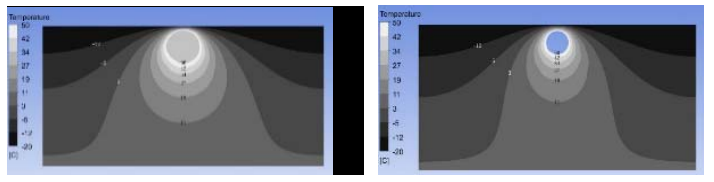

(c)与常温流体 1:2 混合

(d) 与常温流体 1:4 混合

(c) 1:2 mixing of cold and hot fluid (d) 1:4 mixing of cold and hot fluid

图 9 不同比例冷热流体混合时土壤内的温度分布

Fig.9 Temperature distribution in soil when cold and hot fluids are mixed in different proportions
由图 8 可以看出, 随着常温流体混合比例的增加, 入口温度大幅下降, 但由于流速提高, 管道出口流体 的温度几乎没有降低。由图 9 可以看出随着常温流体 混合比例的增加, 土壤中温度高于 $50^{\circ} \mathrm{C}$ 区域在逐渐减 少。

\section{3 包覆保温材料对伪装效果的影响分析}

采用不同导热系数的保温材料对管道进行包覆, 对其土壤表面温度、土壤温度分布进行了计算, 其结 果如图 10、图 11、图 12 所示。

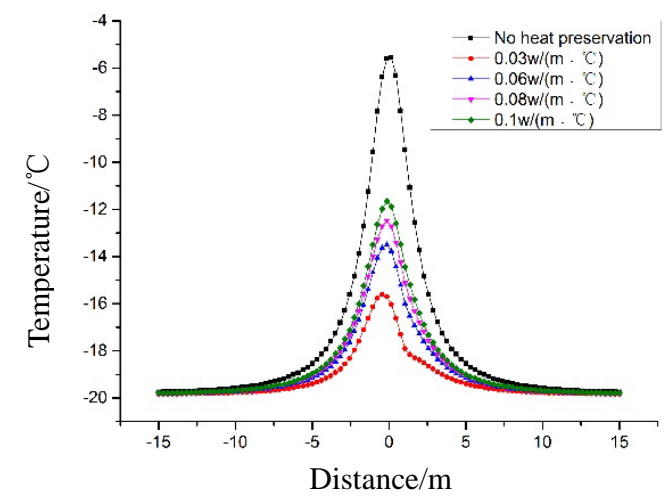

图 10 直埋热管道包覆不同保温材料时的地表温度分布

Fig.10 Surface temperature distribution of direct buried heat pipelines coated with different thermal insulation materials

由图 10 可以看出, 保温层对直埋热管道的地温 温度影响较大。随着导热系数的减小, 地表最大温差 $\Delta t$ 、地表温差大于 $4^{\circ} \mathrm{C}$ 宽度、土壤中温度高于 $50^{\circ} \mathrm{C}$ 区 域均在减少, 但管道出口温度在逐渐上升。对地表最 大温差 $\Delta T$ 和保温层导热系数 $k$ 的关系进行了拟合, 得 出以下拟合公式。

$$
\Delta T=0.5292 k^{3}-4.2275 k^{2}+11.463 k-3.69
$$

由图 11 可以看出, 随着保温层导热系数的减小, 管道出口流体温度逐渐上升。由图 12 可以看出, 随 着保温层导热系数的减小, 土壤中温度高于 $50^{\circ} \mathrm{C}$ 区域 在逐渐减少。

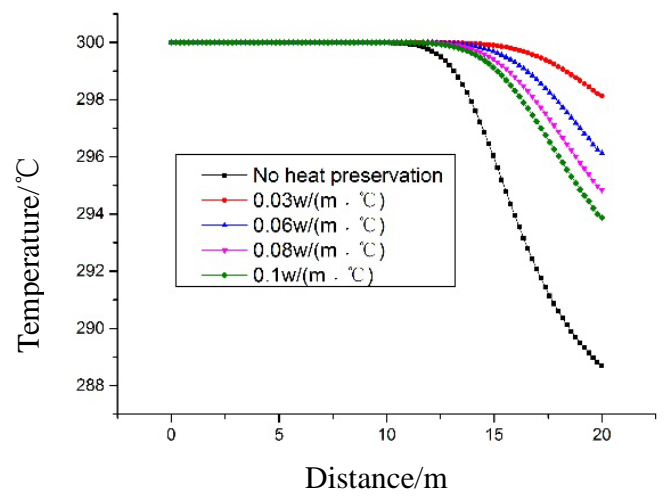

图 11 直埋热管道包覆不同保温材料时的流体温度分布

Fig.11 Fluid temperature distribution of direct buried heat pipe coated with different thermal insulation materials 


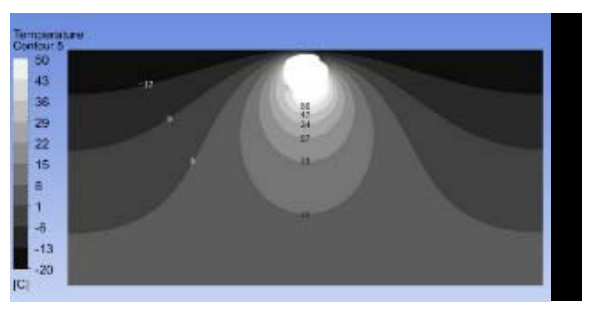

(a) 导热系数 $0.03 \mathrm{~W} /\left(\mathrm{m} \cdot{ }^{\circ} \mathrm{C}\right)$

(a) Thermal conductivity $0.03 \mathrm{~W} /\left(\mathrm{m} \cdot{ }^{\circ} \mathrm{C}\right.$ )

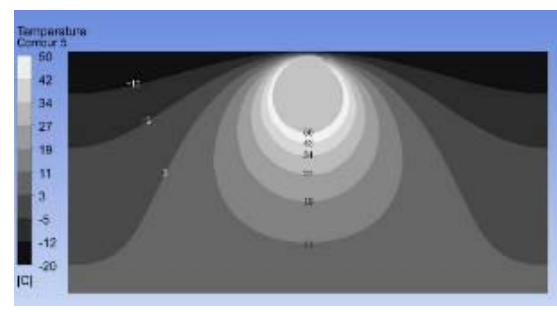

(b) 导热系数 $0.08 \mathrm{~W} /\left(\mathrm{m} \cdot{ }^{\circ} \mathrm{C}\right)$

(b) Thermal conductivity $0.08 \mathrm{~W} /\left(\mathrm{m} \cdot{ }^{\circ} \mathrm{C}\right)$

图 12 不同保温材料时土壤内的温度分布

Fig.12 Temperature distribution in soil under different insulation materials

\section{4 结论}

通过以上的计算及分析, 可以得出以下结论：(1) 采用增加管道埋深、冷热流体混合降温、管道包覆保 温材料 3 种措施均能有效控制地表温差, 并满足直埋 热管道的红外伪装要求; (2)增加管道埋深, 地表辐射 温差逐渐减小, 但土壤中的温度随之增加, 这对植物 伪装是不利的, 所以在有植物伪装时可以考虑增加保 温层, 以控制土壤中的温度; (3)采用增加管道埋深、 管道包覆保温材料这两种措施对管道出口温度的影 响不大, 冷热流体混合能大幅降低管道的出口温度, 但也远远满足不了红外伪装的要求, 因此对直埋热管 道要在出口处进行隔热、降温等措施进行红外伪装: (4)在实际工程实践中, 应综合考虑增加管道埋深、采
取冷热流体混合降温、管道包覆保温材料 3 种措施进 行热红外伪装，消除直埋热管道的红外线性特征。

\section{参考文献:}

[1] 周鹏, 王明时, 陈书旺, 等. 用红外成像法探测埋地输油管道[J]. 石油 学报, 2006(9): 127-130.

ZHOU Peng, WANG Minshi, CHEN Shuwang. Detection of buried oil pipelines by infrared imaging[J]. Petroleum Journal, 2006(9): 127-130.

[2] 张静, 吴明. 用有限元法计算埋地热油管道土壤温度场[J]. 辽宁石油 化工大学学报, 2004(6): 38-41.

ZHANG Jing, WU Ming. Calculation for Soil Temperature Field of Underground Oil Pipeline by Finite Element Method[J]. Journal of Lianning University of Petroleum Chemical Technology, 2004(6): 38-41.

[3] 王常斌, 徐洋, 赵艳红, 等. 埋地热油管道沿程温降的数值模拟 [J]. 管 道技术与设备, 2012(1): 15-17.

WANG Changbin, XU Yang, ZHAO Yanhong. Numerical Simulation of Buride Hot Oil Pipelines[J]. Pipeline Technique and Equipment, 2012(1): 15-17.

[4] 安家荣, 李健, 魏伟, 等. 基于 SPS 软件的埋地热油管道停输温降分 析[J]. 管道技术与设备, 2014(3): 4-6.

AN Jiarong, LI Jian, WEI Wei. Analysis of the Temperature Drop during the Process of Shutdown of Buried Hot Oil Pipeline Based on SPS[J]. Pipeline Technique and Equipment, 2014(3): 4-6.

[5] 胡金文, 马贵阳, 高岩, 等. 不同季节埋地热油管道周围土壤温度场数 值模拟[J]. 当代化工, 2011, 40(6): 619-614.

HU Jinwen, MA Guiyang, GAO Yan. Numerical Simulation of Soil Temperature Filed Around Buried Hot Oil Pipelines in Different seasons[J]. Contemporary Chemical Industry, 2011, 40(6): 619-614.

[6] 赵永涛. 埋地热油管道周围温度场数值模拟 [J]. 新疆石油天然气, 2007, 3(1): 80-86.

ZHAO Yongtao. Numerical Simulation of the Temperature Distribution Around the Underground Heated Oil Pipeline[J]. Journal of Chengde Petroleum College, 2007, 3(1): 80-86. 